\title{
Aplicación del modelo de daño de Gurson-Tvergaard-Needleman (GTN) para la simulación del ensayo miniatura de punzonado sobre probetas prefisuradas
}

\author{
I.I. Cuesta*, J.M. Alegre* y H. Barbáchano*
}

\begin{abstract}
Resumen
El ensayo miniatura de punzonado (SPT) constituye una alternativa factible para la determinación de las propiedades mecánicas, en aquellos casos en los que no se dispone de material suficiente para la realización de ensayos convencionales. Actualmente, existe un gran interés en la utilización de este tipo de ensayo, sobre probetas prefisuradas (P-SPT), para la determinación de las propiedades a fractura del material. En el caso de que el comportamiento del material sea dúctil, el modelo de daño de Gurson-Tvergaard-Needleman (GTN) permite, en base a una serie de parámetros constitutivos, reproducir el comportamiento del material hasta su rotura. Los parámetros constitutivos tienen en cuenta la nucleación, crecimiento y coalescencia de microhuecos, como mecanismos de evolución del daño en materiales dúctiles. El objetivo del presente trabajo es el desarrollo de una metodología para la identificación de dichos parámetros constitutivos, a partir del ajuste numérico de los resultados experimentales obtenidos con los ensayos P-SPT.
\end{abstract}

Palabras clave

Ensayo miniatura de punzonado; Probeta prefisurada; Modelo de daño de Gurson-Tvergaard-Needleman; Parámetros de daño; Fractura dúctil.

\section{Use of the Gurson-Tvergaard-Needleman (GTN) damage model to simulate small punch test on pre-cracked specimens}

\begin{abstract}
Small Punch Test (SPT) is a feasible alternative to determine the mechanical properties in those cases where there is not enough material for conducting conventional tests. Nowadays, there is a great interest in using this test on precracked specimens (P-SPT) in order to determine the fracture properties of the material, as well. In the case of ductile material behaviour, the Gurson-Tvergaard-Needleman (GTN) damage model can be used, based on a set of constitutive parameters, to reproduce the material behaviour up to final fracture. These constitutive parameters take into account the nucleation, growth and coalescence of microvoids as mechanisms of damage evolution in ductile materials. The aim of this paper is to develop a methodology for the identification of these constitutive parameters, based on the numerical adjustment of the experimental results obtained from the P-SPT tests.
\end{abstract}

Keywords

Small punch test; Pre-cracked specimen; Gurson-Tvergaard-Needleman damage model; Damage parameters; Ductile fracture.

\section{INTRODUCCIÓN}

El ensayo miniatura de punzonado o Small Punch Test (SPT) fue desarrollado por Baik et al..$^{[1]}$, en la década de los 80 , en el campo nuclear, con el objetivo principal de obtener las propiedades mecánicas de material irradiado de componentes en servicio, ya que no se disponía de material suficiente para la realización de ensayos convencionales. El ensayo consiste, básicamente, en un punzonado sobre una probeta, mediante un punzón de gran rigidez, estando la periferia de la probeta empotrada por una matriz, como se puede observar en la figura 1. Estas probetas son de reducido tamaño, generalmente cuadradas de dimensiones $10 \times 10 \times 0,5 \mathrm{~mm}$, por lo que el ensayo SPT puede ser considerado como un ensayo no destructivo del componente en servicio analizado.

El resultado del ensayo es una curva carga-desplazamiento, de la que pueden ser extraídos ciertos parámetros como la carga de fluencia, la carga

\footnotetext{
* Departamento de Ingeniería Civil, Universidad de Burgos, E.P.S., C/ Villadiego, s/n, 09001 Burgos. e-mail: iicuesta@ubu.es; jalegre@ubu.es.
} 


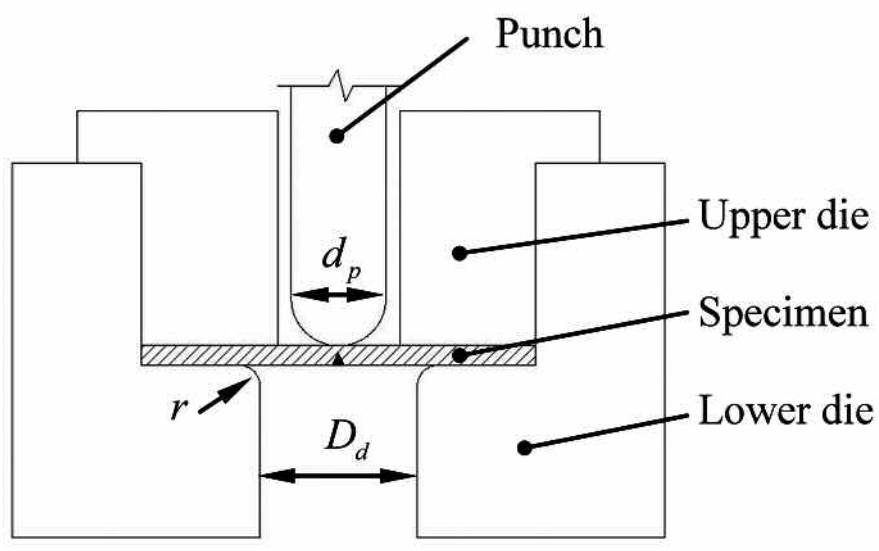

a)

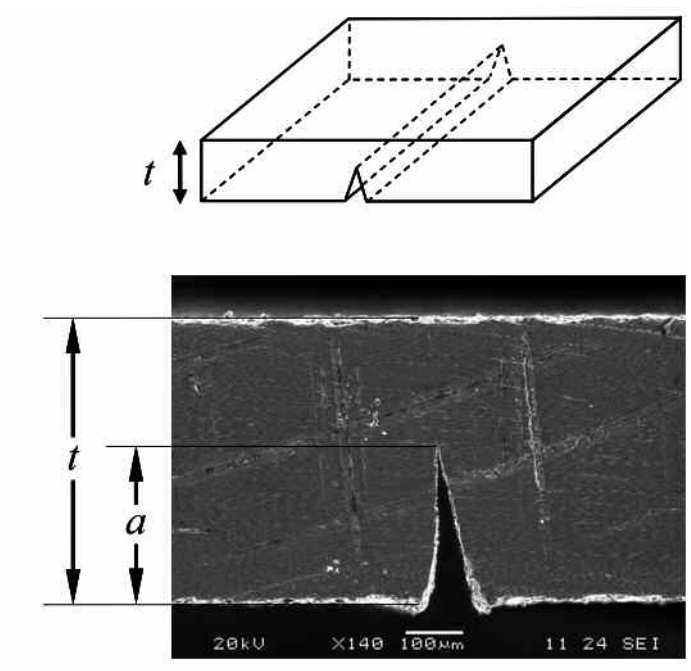

b)

Figura 1. Small Punch Test: a) esquema y b) probeta SPT prefisuradas por láser.

Figure 1. Small Punch Test: a) testing device and b) laser pre-cracked SPT specimen.

máxima, el desplazamiento en el instante de fallo o el área bajo la curva, entre otros. Los primeros investigadores en emplear estos parámetros fueron Mao et al. ${ }^{[2}$ y 3$]$, desarrollando una serie de expresiones empíricas para extraer las propiedades mecánicas y de fractura del material analizado a partir el SPT. En los primeros años de la década de los 90, Misawa et al. ${ }^{[4-8]}$ se valieron de los avances realizados por Mao et al. [2 y 3$]$ para alcanzar resultados satisfactorios en sus investigaciones. Estos estudios conforman los primeros intentos de obtener las propiedades a fractura de un material a partir del SPT sirviendo a otros autores, en multitud de ocasiones en años posteriores, como base sólida de sus investigaciones ${ }^{[9-13]}$. Hay que hacer notar que la gran mayoría de estos trabajos han sido llevados a cabo sobre probetas convencionales de SPT, siendo muy pocos los autores que han utilizado probetas prefisuradas ${ }^{[14-16]}$. A priori, estas probetas prefisuradas, por tener mecanizada una grieta inicial, podrían suponer una mejor opción a la hora de estimar las propiedades a fractura.

Una de las técnicas más utilizadas, actualmente, para el estudio de la fractura de materiales metálicos es la aproximación local de la fractura. Esta aproximación pretende analizar en profundidad el mecanismo de rotura y la relación que existe entre la microestructura y la composición del material (aspectos metalúrgicos) con la evolución del daño hasta el instante de fallo. En el caso de que el comportamiento del material sea dúctil, el modelo de daño de Gurson-Tvergaard-Needleman permite, en base a la utilización de una serie de parámetros cons- titutivos, reproducir el comportamiento del material hasta su rotura. Estos parámetros hacen referencia a la nucleación, crecimiento y coalescencia de microhuecos, como mecanismos de evolución del daño en materiales dúctiles. Uno de los aspectos más desconocidos de la aplicación de este modelo de daño es la relación que existe entre los parámetros matemáticos del modelo y la microestructura/ composición del material.

El objetivo del presente trabajo es el desarrollo de una metodología para la correcta identificación de los parámetros del modelo de daño de GTN, a partir del ajuste de la curva experimental obtenida en el SPT sobre probetas prefisuradas. Debido a la propia formulación del modelo, los parámetros que lo definen comienzan a tener importancia en diferentes instantes, a lo largo del ensayo. Este hecho es ventajoso ya que permite un cierto desacoplamiento a la hora de identificarlos. La metodología propuesta, a pesar de haber sido desarrollada a partir de los resultados obtenidos con probetas de un tipo de acero inoxidable, en principio, se podría utilizar de igual forma, en el caso de trabajar con otros tipos de aceros.

\section{MATERIAL}

El material elegido en la presente investigación es un acero inoxidable martensítico endurecido por precipitación (15,5 $\mathrm{PH})$, en este caso, condición 1025 (calentamiento de $4 \mathrm{~h}$ a una temperatura de 
$551,7^{\circ} \mathrm{C} \pm 8^{\circ} \mathrm{C}$ y enfriamiento al aire). La tabla I recoge la composición química de este acero.

La caracterización del comportamiento del material en tracción se ha efectuado mediante el ensayo de probetas convencionales siguiendo la norma ASTM E 8M ${ }^{[17]}$, ajustándolo a una ecuación, tipo Ramberg Osgood ${ }^{[18]}$. Comúnmente, en los aceros se acepta que la deformación plástica comienza a tenerse en cuenta cuando esta alcanza un valor del 0,2 \%, con lo que el modelo de Ramberg Osgood puede ser simplificado según la expresión (1).

$$
\varepsilon=\frac{\sigma}{E}+0.002 \cdot\left(\frac{\sigma}{\sigma_{0.2}}\right)
$$

donde, $\sigma_{0,2}$ es el límite elástico para una deformación plástica del 0,2 \% y $n$ es el parámetro de endurecimiento ${ }^{[18]}$. De esta manera, queda definido el comportamiento elastoplástico del material, a través de los parámetros elásticos: módulo de Young $(E)$ y coeficiente de Poisson $(v)$ y de los parámetros plásticos: $\sigma_{0,2}$ y n, cuyos valores se recogen en la tabla II.

Tabla I. Composición química del acero $15.5 \mathrm{PH}$

Table I. Chemical composition of the 15.5 PH stainless steel

\begin{tabular}{cc}
\hline Elemento & $\begin{array}{c}\text { Composición } \\
\text { (\% en peso) }\end{array}$ \\
\hline $\mathrm{C}$ & 0,043 \\
$\mathrm{Mn}$ & 0,810 \\
$\mathrm{Si}$ & 0,280 \\
$\mathrm{Ni}$ & 5,000 \\
$\mathrm{Cr}$ & 14,660 \\
$\mathrm{Cu}$ & 3,060 \\
$\mathrm{Nb}$ & 0,380 \\
$\mathrm{P}$ & 0,017 \\
$\mathrm{~S}$ & 0,001 \\
\hline
\end{tabular}

Tabla II. Parámetros elastoplásticos del acero $15.5 \mathrm{PH}$

Table II. Elastoplastic parameters of the 15.5 PH stainless steel

\begin{tabular}{cccc}
\hline $\boldsymbol{E}(\mathbf{M P a})$ & $v$ & $\sigma_{0.2}(\mathbf{M P a})$ & $\boldsymbol{n}$ \\
\hline 200.000 & 0,3 & 1100 & 38,8 \\
\hline
\end{tabular}

\section{MÉTODO EXPERIMENTAL}

En el desarrollo del presente trabajo se han utilizado probetas SPT prefisuradas, de dimensiones $20 \times 20 \times 1$ $\mathrm{mm}$, con una fisura inicial de tipo longitudinal no pasante, como se puede ver en la figura 1. Las probetas utilizadas en la presente investigación están escaladas al doble del tamaño de las probetas SPT convencionales $(10 \times 10 \times 0,5 \mathrm{~mm})$, para poder analizar con mayor precisión el efecto de diferentes longitudes de prefisuración. Para la creación de la fisura/entalla inicial se han empleado dos técnicas diferentes, el micromecanizado de alta precisión y la microfisuración por láser.

En el micromecanizado se ha empleado una herramienta en forma de $\mathrm{V}$ con ángulo de $37^{\circ}$ y radio de redondeo en la punta de $100 \mu \mathrm{m}$. La profundidad de fisura deseada se obtiene aplicando la herramienta, longitudinalmente, a partir de la cara inferior, desde el centro de un lado de la probeta hacia el centro del lado opuesto. La geometría de la fisura inicial que se obtiene con esta técnica se puede ver en la figura 2, la cuál reproduce, fielmente, la forma de la herramienta con la que se ha mecanizado. Cabe destacar el buen acabado superficial de la fisura y la homogeneidad conseguida de la profundidad de fisura a lo largo de toda su longitud.

Por otro lado, en la microfisuración por láser, tras una calibración inicial de la potencia para conseguir la profundidad de fisura deseada, el láser es aplicado en 25 pasadas, en forma de haz de pulsos (Nd:YAG) de $30 \mu \mathrm{m}$ de diámetro ( $50 \mathrm{~W}$ y 1,064 nm), trabajando a una frecuencia de $7.500 \mathrm{~Hz}$ y con un desplazamiento

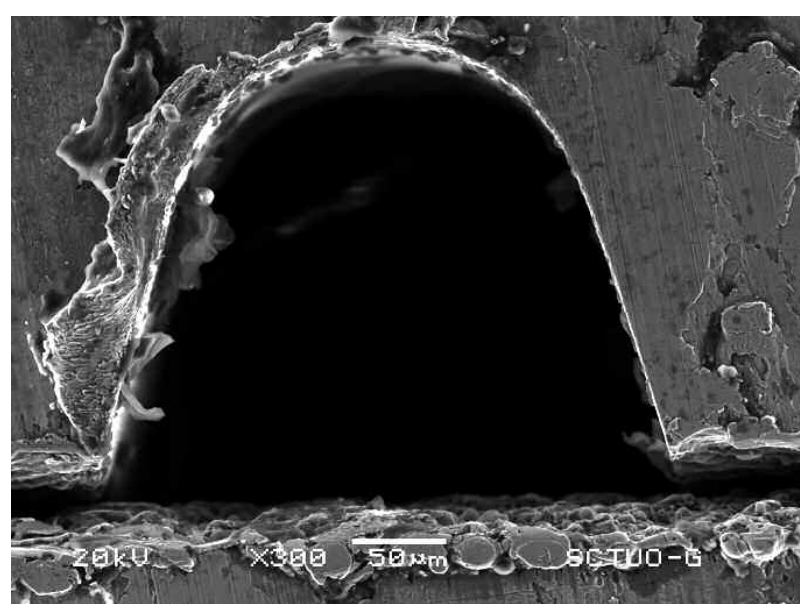

Figura 2. Sección de la fisura inicial obtenida con el micromecanizado de alta precisión.

Figure 2. Cross section of the crack-like notch obtained by high precision micromachining. 
lineal de $15 \mathrm{~mm} / \mathrm{s}$. Al igual que en la técnica anterior, la fisura se realiza de forma longitudinal, a partir de la cara inferior, desde el centro de un lado de la probeta hacia el centro del lado opuesto. En la figura 3 se muestra la sección de la fisura inicial obtenida con esta técnica, donde se puede observar que, en este caso, es mucho más aguda y la punta de fisura es afilada, asemejándose más a una fisura perfecta sin redondeo en la punta. Para comprobar el alcance de la zona afectada térmicamente (ZAT) por el láser se han realizado una serie de huellas de microdureza en las proximidades de la grieta (Fig. 4). Para este material, se

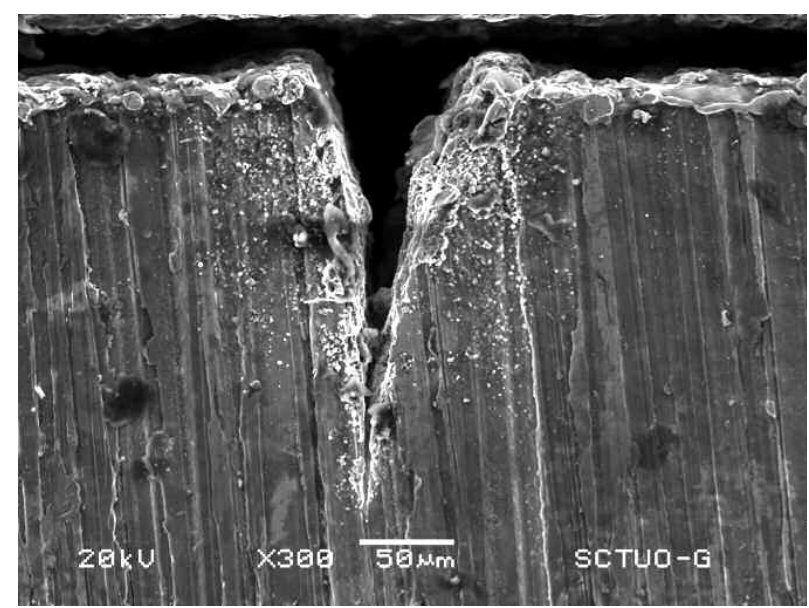

Figura 3. Sección de la fisura inicial obtenida con la microfisuración por láser.

\section{Figure 3. Cross section of the crack obtained by laser-induced micromachining.}

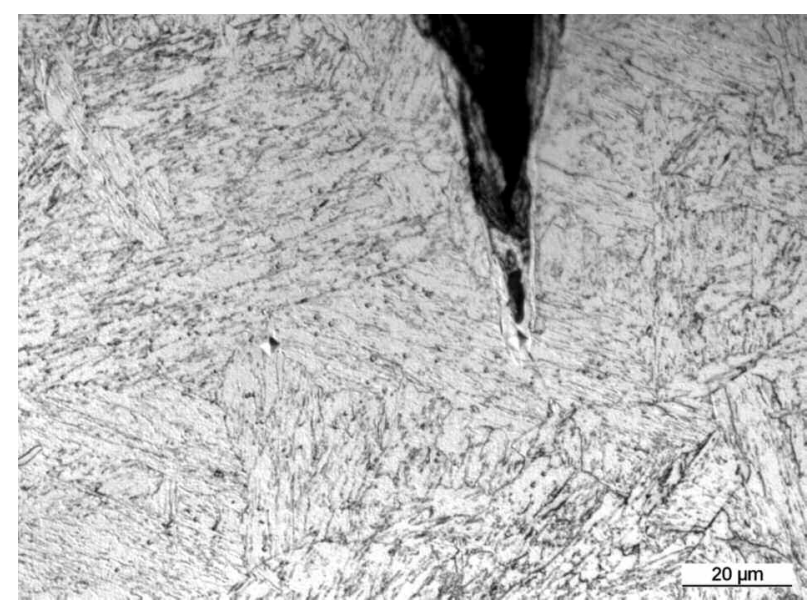

Figura 4. Huellas de dureza en las proximidades de la fisura generada con microfisuración por láser.

Figure 4. Indentations in the close area of the crack generated by laser-induced microcracking. observa una diferencia poco significativa en el tamaño de las huellas (HV $=410 \pm 1$, bajo una carga de 27 $\mathrm{mN}$ ), lo que indica que la ZAT puede ser muy pequeña. Sin embargo, este análisis debe ser efectuado en el caso de la aplicación a otros materiales, con mayores contenidos en carbono y más sensibles a la variación térmica generada por el láser.

Cabe destacar, que esta técnica presenta el inconveniente de conseguir un frente de fisura más irregular (Fig. 5), donde la profundidad de fisura es, ligeramente, variable en longitud ya que los pulsos láser van dejando picos y valles, a lo largo del extremo de la fisura, conforme ha ido avanzando, como se puede ver en el detalle de la figura 5. La variabilidad de la profundidad de fisura se ha estimado entorno al $10 \%$, con esta técnica, mientras que con el micromecanizado de alta precisión la profundidad es, prácticamente, constante y es, por ello, que los ensayos realizados requerirán de una medida posterior de la fisura obtenida, mediante técnicas de microscopía óptica de barrido (SEM). En cualquier caso, las curvas cargadesplazamiento obtenidas a partir de probetas SPT
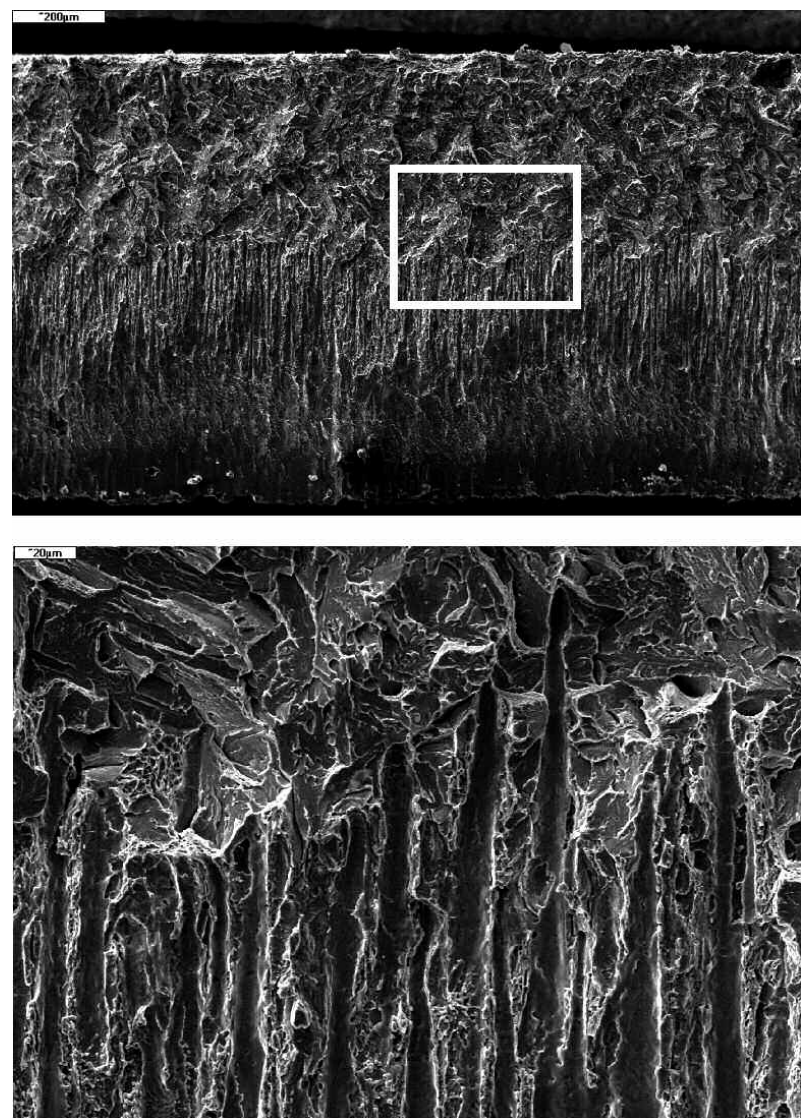

Figura 5. Frente de fisura obtenido con la microfisuración por láser.

Figure 5. Crack front obtained by laser-induced micromachining. 
prefiguradas, con cualquiera de estas dos técnicas, son semejantes, siempre y cuando la profundidad de la fisura inicial tenga el mismo valor en las probetas analizadas.

Independientemente de la técnica utilizada en la prefisuración de la probeta, el SPT puede ser llevado a cabo sobre probetas con diferentes profundidades de fisura $(a)$. Dicha profundidad se puede hacer adimensional con respecto al espesor de probeta $(t)$ obteniendo, de esta manera, diferentes valores de la relación $a / t$, siendo los más habituales valores teóricos de $a / t$ entre 0,2 y 0,5 .

Para la realización del ensayo se ha utilizado un diámetro del punzón, $d_{p}=5 \mathrm{~mm}$, una matriz inferior de diámetro, $D_{d}=8 \mathrm{~mm}$, y un radio de acuerdo de la matriz inferior, $r=1 \mathrm{~mm}$. El ensayo se ha realizado a temperatura ambiente. La velocidad de ensayo ha sido $\mathrm{v}=0,2 \mathrm{~mm} / \mathrm{min}$.

\section{SIMULACIÓN NUMÉRICA DEL SPT}

El software utilizado para la simulación ha sido ABAQUS Explicit. Debido a la simetría que presenta el SPT, se ha modelizado un cuarto de la geometría, utilizando los planos $\mathrm{YZ}$ y XY como planos de simetría, como se observa en la figura 6 . El punzón y las matrices superior e inferior se han considerado cuerpos rígidos ya que sus deformaciones durante el ensayo son, prácticamente, despreciables en comparación con las que se producen en la probeta. La probeta se ha considerado como un cuerpo deformable elastoplástico y ha sido modelada con elementos hexaédricos de ocho nodos.

El coeficiente de fricción $(\mu)$ entre los diferentes cuerpos en el contacto es un parámetro a tener en cuenta en la simulación ${ }^{[19]}$. Como el contacto es aceroacero se ha tomado un valor $\mu=0,18$. Las dimensiones geométricas son las mismas que las del ensayo experimental, siendo el espesor de la probeta $1 \mathrm{~mm}$.

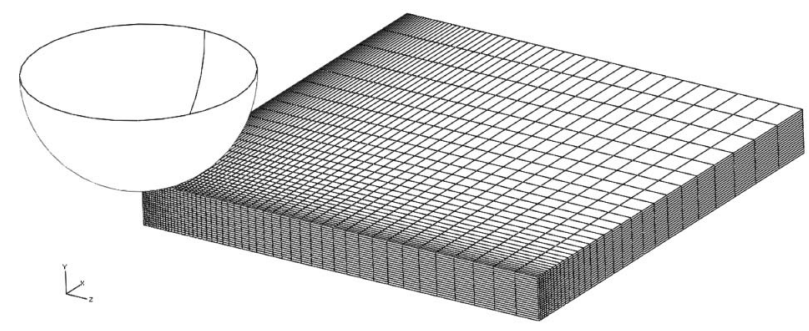

Figura 6. Mallado de un cuarto de probeta P-SPT.

Figure 6. A quarter mesh of the P-SPT specimen.
Se han utilizado las propiedades elásticas y plásticas del material (Tabla II) obtenidas a partir de los datos experimentales de la curva tensión-deformación. El modelo de daño implementado ha sido el modelo de GTN. Todas las simulaciones se han realizado con el mismo tamaño de elemento (elementos hexaédricos de 0,05 mm de lado), en la zona central de la probeta, que es donde se produce el inicio del fallo. Este tamaño de malla debe acompañar, siempre, a los resultados de los parámetros constitutivos del material obtenidos ${ }^{[20]}$.

\section{MODELO DE DAÑO}

\subsection{Ecuación constitutiva}

El modelo original de Gurson ${ }^{[21}$ y 22] venía definido por una ecuación constitutiva que incluía un parámetro de daño denominado porosidad o fracción volumétrica de microhuecos $(f)$, que varía entre 0 (material sin daño) y 1 (material completamente dañado). El mayor inconveniente de este modelo era la incapacidad de predecir el fallo final causado por la coalescencia de microhuecos en la fractura dúctil. Para solventar este problema, Tvergaard y Needleman ${ }^{[23-25]}$ modificaron el modelo original, obteniendo como ecuación constitutiva la expresión (2), que define el modelo de daño de GTN, utilizado en este trabajo:

$$
F=\left(\frac{\bar{\sigma}}{\sigma_{y}}\right)^{2}+2 q_{1} f^{*} \cosh \left(\frac{q_{2} \sigma_{k k}}{2 \sigma_{y}}\right)-\left[1+\left(q_{1} f^{*}\right)^{2}\right]=0
$$

donde, $\sigma_{k k}$ es el primer invariante del estado tensional, $\bar{\sigma}$ es la tensión equivalente de Von Mises y $\sigma_{y}$ es la tensión de fluencia de la matriz. Los coeficientes propuestos por Tvergaard y Needleman para amplificar el efecto de la tensión hidrostática en todos los niveles de deformación fueron $q_{1}$ y $q_{2}$. Para el caso de los metales ${ }^{[26 \text { y } 27]}$, es razonable tomar un valor constante de $q_{1}=1,5$ y $q_{2}=1$. Finalmente, el parámetro $f^{*}$, definido por la expresión (3), es la porosidad modificada y se introduce en el modelo para poder reproducir el decrecimiento de la capacidad de carga, en los instantes en los que se alcanza la coalescencia de microhuecos.

$$
f^{*} \begin{cases}f & \text { si } f \leq f_{c} \\ f_{c}+\delta\left(f-f_{c}\right) & \text { si } f>f_{c}\end{cases}
$$


donde, $f$ es la porosidad y $f$ es la porosidad crítica; este parámetro nos indica el valor para el cuál la coalescencia de microhuecos comienza a tener lugar en el material, con lo que la capacidad resistente desciende notablemente. Por último, $\delta$ es la velocidad de coalescencia que viene dada por la expresión (4).

$$
\delta=\frac{f_{u}^{*}-f_{c}}{f_{F}-f_{c}}
$$

en la que $f_{\mathrm{u}}^{*}=1 / q_{1}$ y $f_{\mathrm{F}}$ es la fracción volumétrica de microhuecos en el fallo. Una vez que se inicia la coalescencia de microhuecos, la fracción volumétrica de los mismos sigue aumentando hasta alcanzar el valor de $f_{F}$, momento en el que se produce la rotura del material. Una vez fijado el valor $\operatorname{de} f_{\mathrm{u}}^{*}$, los valores $\operatorname{de} f_{\mathrm{c}} \mathrm{y} f_{\mathrm{F}}$ van a ser los que gobiernen la velocidad de coalescencia $(\delta)$.

\subsection{Evolución de la porosidad}

Las ecuaciones anteriores establecen el comportamiento del material cuando se considera un valor de porosidad específico. Sin embargo, conforme se incrementa el nivel de deformación en el material, la porosidad también se incrementa, debido a dos fenómenos: por un lado, los microhuecos existentes van creciendo gradualmente y, por el otro, nuevos microhuecos se generan en el material como resultado del aumento de la deformación. En consecuencia, si se controlan la nucleación y el crecimiento en función de la deformación, el incremento de la porosidad viene establecido por la expresión (5).

$$
\begin{aligned}
\dot{f}= & \dot{f}_{\text {crecimiento }}+\dot{f}_{\text {nucleacion }} \\
\dot{f}_{\text {crecimiento }} & =(1-f) \dot{\varepsilon}_{k k}^{p} \\
\dot{f}_{\text {nucleacion }} & =\frac{f_{N}}{S \sqrt{2 \pi}} \exp \left[-\frac{1}{2}\left(\frac{\varepsilon_{m}^{p}-\varepsilon_{n}}{S}\right)^{2}\right] \dot{\varepsilon}_{m}^{p}
\end{aligned}
$$

En la expresión anterior, el crecimiento de los microhuecos existentes está controlado por la traza del tensor de deformación plástica $\dot{\varepsilon}_{k k^{*}}^{p}$. En el instante inicial, previo a la realización del ensayo, la porosidad del material queda definida por la fracción volumétrica de microhuecos inicial, $f_{0}$. Existen varias técnicas para poder estimar este parámetro, una de ellas es el análisis SEM del material sin daño, lo cuál puede resultar ciertamente complejo, en algunas ocasiones. Una alternativa posible es la utilización de la fórmula propuesta por Franklin ${ }^{[28]}$, definida a partir de la composición química del material analizado (Exp. 6), en concreto, del porcentaje de inclusiones de manganeso y azufre. Estas inclusiones pueden ser consideradas como microhuecos iniciales ya que concentran el temprano crecimiento de los primeros microhuecos.

$$
f_{0} \approx 0.054\left(S(\%)-\frac{0.001}{M n(\%)}\right)
$$

Introduciendo los datos de la tabla I, se obtiene, en este caso, un valor de $f_{0} \approx 0$, ya que los términos del interior del paréntesis de la expresión (6), prácticamente, se anulan entre sí, resultando, finalmente, un valor poco significativo. Este hecho es indicativo de que el acero 15,5 $\mathrm{PH}$ puede ser considerado como un acero denso, es decir, que los microhuecos que producirán el fallo se generarán por nucleación, a lo largo del proceso de deformación de la probeta. Dicha nucleación de microhuecos depende de los siguientes parámetros:

- $\varepsilon_{n}$ es la deformación media para la nucleación. En las zonas de material en las que se alcanza este valor de deformación comienza la nucleación de microhuecos y su posterior crecimiento si la deformación aumenta.

- S es la desviación estándar del parámetro $\varepsilon_{n}$, ya que este sigue una distribución normal en el modelo de daño. El valor $S$ de será pequeño si el material se considera homogéneo, mientras que tomará un valor elevado si el tamaño de las partículas de la segunda fase del material varia mucho. De acuerdo con varios estudios realizados sobre este tipo de materiales ${ }^{[29]}$, se puede asumir un valor típico de $S=0,01$.

- $f_{\mathrm{N}}$ es la fracción volumétrica de la nucleación de microhuecos. Con este parámetro se controla la fracción volumétrica de microhuecos que se forman cuando se alcanza el valor de $\varepsilon_{n}$, es decir, cuando comienza la nucleación.

\section{INFLUENCIA DE LOS PARÁMETROS DE MATERIAL EN EL SPT}

En el apartado anterior, se ha podido comprobar como la propia formulación del modelo de daño de GTN implica que los parámetros que lo definen comienzan a tener importancia, en diferentes instantes, a lo largo del proceso de deformación de la probeta. Este hecho es ventajoso, ya que permite un cierto desacoplamiento a la hora de identificar los parámetros del modelo de daño, a través del estudio de las diferentes regiones de la curva carga-desplazamiento obtenida en el SPT. 
La figura 7 presenta las curvas típicas carga-desplazamiento obtenidas en el SPT con probetas prefisuradas, en función de si la relación $a / t$ es mayor o menor de 0,5, para el material considerado en el presente trabajo. Analizando la forma de la curvas, es posible discernir diferentes regiones en cada una de ellas e identificar la influencia que los parámetros elastoplásticos del material $\left(E, v, \sigma_{0.2}, n\right)$ y los parámetros de evolución del daño $\left(\varepsilon_{n}, f_{N}, f_{c}, f_{F}\right)$ tienen sobre dichas regiones. Para una mayor comprensión de estas curvas, también, se han representado las obtenidas en un ensayo SPT sin fisura y en la simulación de una fisura completamente pasante, es decir, como si estuviese formada por dos mitades unidas sólo por las fuerzas de contacto (probeta rota en dos mitades).

Para la curva correspondiente a una relación, la región I correspondería al comportamiento puramente elástico de la probeta. En esta región, tanto la probeta como el utillaje se reposicionan ligeramente y se produce el fenómeno de indentación. Los únicos parámetros que tienen influencia en esta región son $E$ y $v$.

La región II correspondería con la transición elastoplástica, ya que en algunas zonas de la probeta comienza a predominar la deformación plástica, especialmente, la zona en contacto con el punzón. Los parámetros $\sigma_{0.2}$ y $n$ son los que definen el comportamiento de esta región. En la región III tiene lugar la plastificación creciente de la probeta y la progresiva abertura de los labios de la fisura, como puede observarse en la secuencia de fractografías de la figura 7 donde, cada una de ellas se corresponde con los círculos negros sobre la curva de la figura. Los mecanismos de daño comienzan a entrar en juego: comienza la nucleación de microhuecos en los puntos de mayor deformación plástica, por lo que los parámetros $\varepsilon_{n}$ y $f_{N}$ empiezan a tener importancia.

La fracción volumétrica de microhuecos crece conforme va aumentando el desplazamiento del punzón; en consecuencia, en el tramo final de esta región, se produce el fenómeno de coalescencia de microhuecos, debido a que la fracción volumétrica de microhuecos alcanza en algunos puntos de la probeta el valor $\operatorname{de} f_{c}$, incluso el valor de $f_{F}$. Este hecho implica el comienzo y propagación de la fisura (Fig. 7 c)), hasta llegar al quiebro que se produce en la curva, entre la región III y IV, en el cuál la fisura se hace pasante a lo largo del espesor de la probeta (Fig. 7 d)).

Con el objetivo de estudiar y clarificar el fenómeno de la iniciación se han efectuado varios ensayos interrumpidos a diferentes niveles de carga. Posteriormente, se han fragilizado con nitrógeno líquido las probetas interrumpidas, para favorecer la rotura, de forma frágil, en dos mitades, facilitando de esta manera el análisis de la evolución del daño

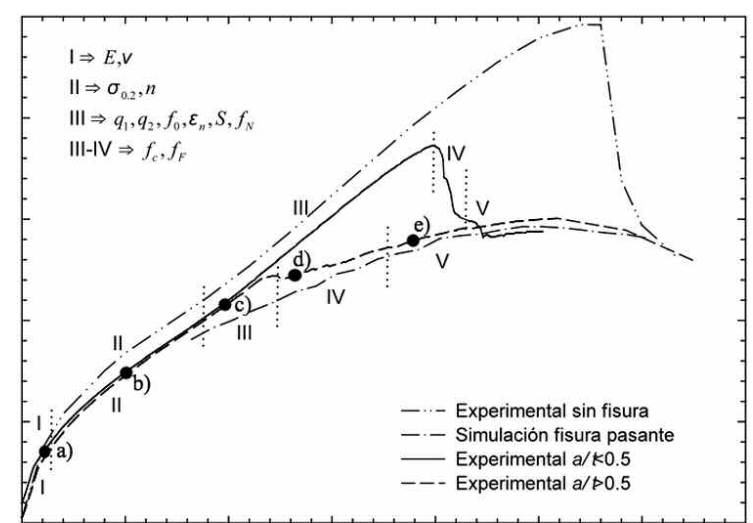

Desplazamiento punzón $(\mathrm{mm})$

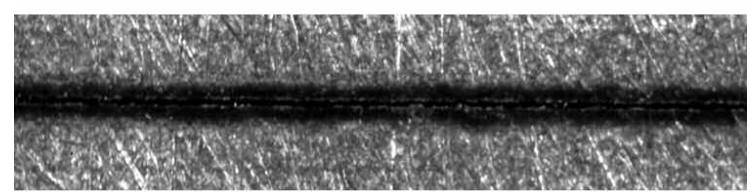

a)

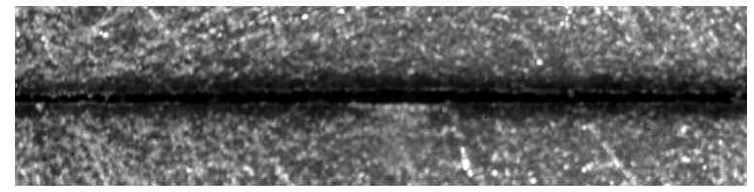

b)

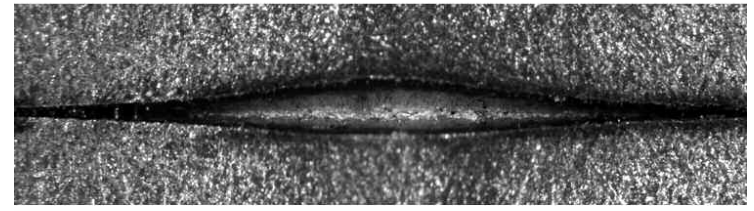

c)

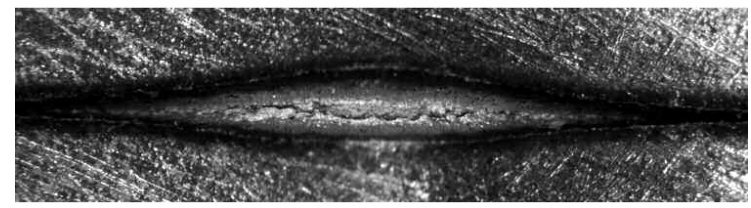

d)

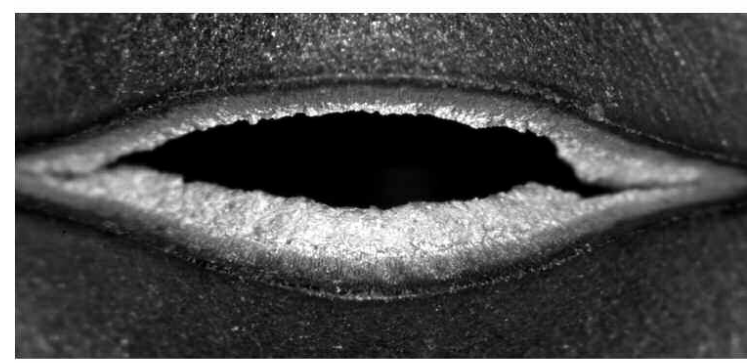

e)

Figura 7. Curvas carga-desplazamiento de probetas prefisuradas SPT y propagación de fisura.

Figure 7. Load-displacement curves of pre-cracked SPT specimens and crack propagation. 
en cada etapa de carga. Dichos ensayos revelan que el inicio de la propagación tiene lugar en el centro del frente de grieta inicial, para un valor aproximado del $85 \%$ de la carga máxima. Conforme va avanzando el ensayo la fisura se convierte en pasante, lo cuál se corresponde con el valor de carga máxima, generando, a partir de ese punto, dos frentes de grieta que avanzarían en sentido opuesto hasta la zona de empotramiento de la probeta ${ }^{[30]}$

La figura 8 muestra la superficie de fractura de la probeta correspondiente a la figura $7 \mathrm{c}$ ), en ella se puede apreciar, claramente, además de las partes correspondientes a la rotura frágil y a la fisura inicial, una parte central, de color claro, correspondiente al inicio de la propagación de la fisura a través de los mecanismos de fractura dúctil. Estos mecanismos se pueden ver, claramente, en la figura 9, en la que se observa como se ha producido la nucleación, crecimiento y coalescencia de microhuecos en la zona de inicio. En esa misma figura se puede apreciar la transición de fractura dúctil a fractura frágil, producida al partir la probeta por fragilización con nitrógeno líquido.

A partir del inicio de la región IV se produce, rápidamente, el desgarro de la probeta hasta alcanzar los bordes de la matriz, instante en el que la probeta tiende a comportarse como dos mitades desacopladas (fisura pasante). Los parámetros y tienen una importancia capital ya que gobiernan la velocidad de rotura de la probeta y, por consiguiente, la forma de la curva en esta región.

La región $\mathrm{V}$, zona de rotura final, puede decirse que comienza cuando la curva carga-desplazamiento se ajusta al comportamiento de dos mitades, como

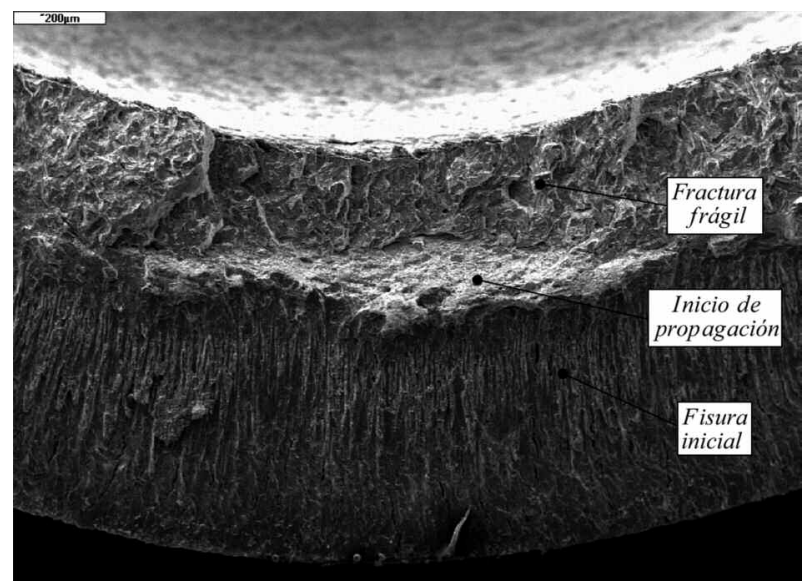

Figura 8. Inicio de propagación de fisura en probetas SPT prefisuradas (75X).

Figure 8. Crack initiation in pre-cracked SPT specimen $(75 X)$.

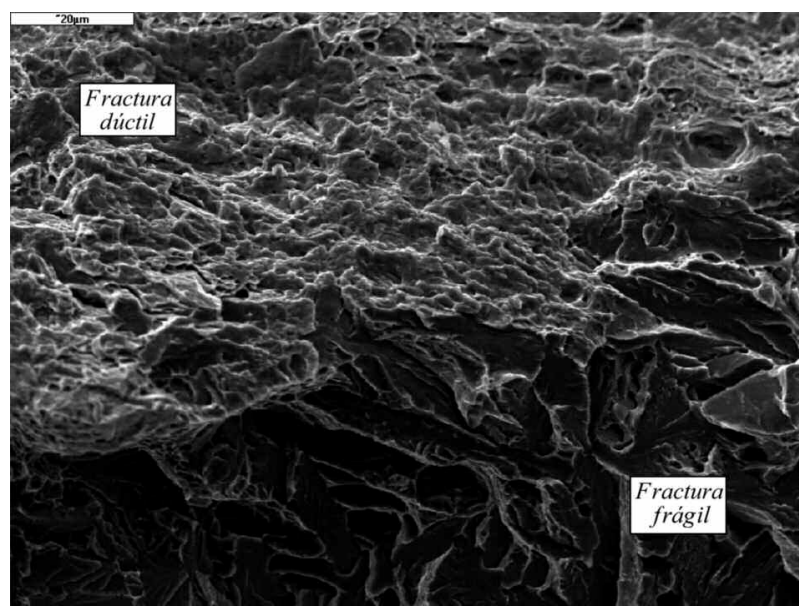

Figura 9. Transición de fractura dúctil a frágil (1.000X).

\section{Figure 9. Ductile to brittle fracture transition} $(1,000 X)$.

se puede ver en la figura 7. En esta zona, el punzón atraviesa por completo la probeta. En los ensayos experimentales no es usual llegar hasta la rotura total de la probeta, ya que el ensayo se suele parar cuando se ha completado la región IV de la curva.

En la curva correspondiente a una relación $a / t$ $<0,5$ se puede llegar a distinguir, de nuevo, hasta cinco regiones más o menos diferenciadas. Las tres primeras se corresponderían con las de la curva anterior, con la salvedad de que la región III es mucho mayor, en este caso, alcanzando la probeta una mayor capacidad de carga al ser mayor el ligamento resistente. A lo largo de la región III, se inicia la fisura y crece hasta hacerse pasante, cuyo valor, en este caso, coincide con el máximo de la curva carga-desplazamiento. En ese instante, se produce un descenso brusco de la capacidad resistente de la probeta. La región IV comprende dicho decremento y, en ella, la fisura propaga en ambos sentidos por todo el espesor hasta llegar a la zona de empotramiento, momento en el que la probeta, al igual que antes, se comporta como dos mitades desacopladas. La última región correspondería al traspaso total del punzón, a través de las dos mitades desacopladas.

\section{METODOLOGÍA PARA LA DETERMINACIÓN DE PARÁMETROS}

Teniendo en cuenta el apartado anterior, la determinación de los parámetros de material a partir de la curva carga-desplazamiento va a ser posible debido a que estos empiezan a tener influencia en diferentes 
regiones de la curva. Los parámetros elastoplásticos $\left(E, v, \sigma_{0.2}, n\right)$ ya han sido determinados de forma directa, mediante los ensayos de tracción, aunque existen métodos para obtener dichos parámetros de forma indirecta, a partir del propio ensayo SPT sin fisura $^{[2}$ y 30$]$.

En consecuencia, únicamente queda por determinar $\varepsilon_{n}, f_{\mathrm{N}}, f_{\mathrm{c}} \mathrm{y} f_{\mathrm{F}}$ todos ellos, parámetros del modelo de daño. El parámetro de inicio de la nucleación, $\varepsilon_{n}$, es habitual considerarlo con un valor de deformación plástica moderado (10\%), para la mayoría de los aceros, $\varepsilon_{n}=0,1^{[26 \text { y } 27]}$. El valor que se obtenga para la tasa de nucleación, $f_{\mathrm{N}}$, ya incluye el efecto del momento de inicio. Es decir, el efecto de una deformación de inicio, $\varepsilon_{n}$ menor, junto con una tasa de nucleación menor, $f_{N}$, equivale a una deformación de inicio, $\varepsilon_{n}$ mayor, junto con una tasa de nucleación mayor, $f_{\mathrm{N}}$.

\subsection{Determinación de $f_{\mathrm{N}}$}

El parámetro $f_{N}$ es el que va a gobernar la forma de la curva, en una parte de la región III (tramo comprendido entre el final de la región II y el instante de inicio de propagación, en torno al 85 \% de la carga máxima), ya que los demás parámetros de los que depende este tramo ya se han determinado y permanecen fijos. Dicho tramo se puede ajustar a una recta $\left(P=m \cdot \Delta p+n_{0}\right)$, por lo que es posible relacionar la pendiente experimental, $m$, con el valor de $f_{\mathrm{N}}$. Para ello, se han realizado una serie de simulaciones numéricas en las que se ha variado $f_{N}$ en un rango de $[0,001$, $0,07]$, que es el rango típico para los aceros $[26$ y 27$]$.

Para obtener el valor experimental de la pendiente se han llevado a cabo tres ensayos sobre probeta prefisurada, con una relación $a / t=0,25$. La elección de un valor de $a / t<0,5$ favorece el ajuste de la región III mediante una recta, ya que el tramo de curva comprendido en esta región es más amplio, como se ha comentado, anteriormente (figura 7).

La figura 10 muestra la variación de la pendiente, $m$, para el rango utilizado de $f_{N}$ en las simulaciones numéricas, el valor de la pendiente, $m$, cambia de 3,31 , para a 2,91, para $f_{N}=0,07$, lo que supone que el efecto de, en el rango estudiado, está entorno a un $12 \%$. A pesar de no ser un valor elevado, es suficiente para poder discernir con claridad el valor óptimo de $f_{\mathrm{N}}$, ya que la dispersión experimental en la región III está entorno al $2 \%$. Se puede observar cómo el valor experimental de $m=3,27$ se encuentra dentro de los límites superior e inferior calculados. Este hecho permite determinar, de manera factible, el valor óptimo de que ajuste el valor experimental de $m$, a partir de la figura 11. En ella, queda

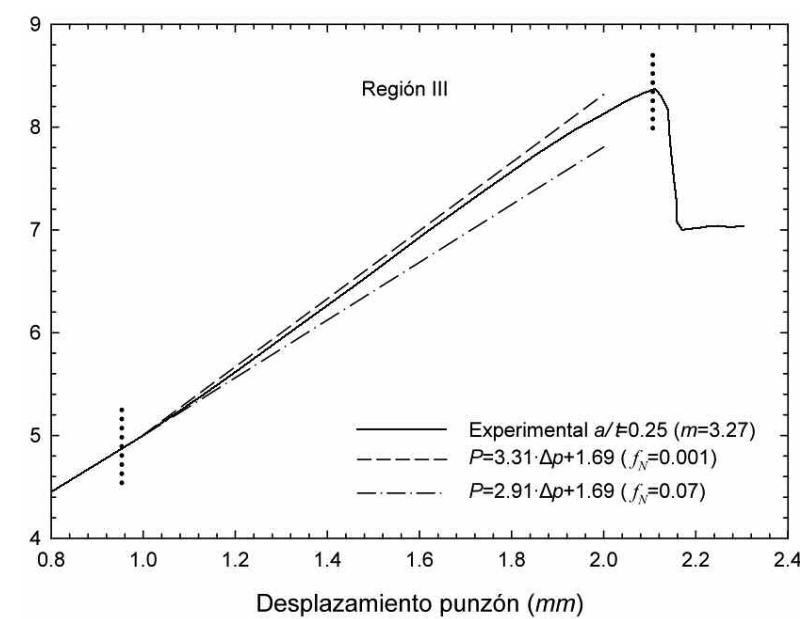

Figura 10. Rectas superior e inferior en la región III obtenidas a partir de la variación de $f_{N}$.

Figure 10. Upper and lower straight lines in stage III obtained with $f_{N}$ variation.

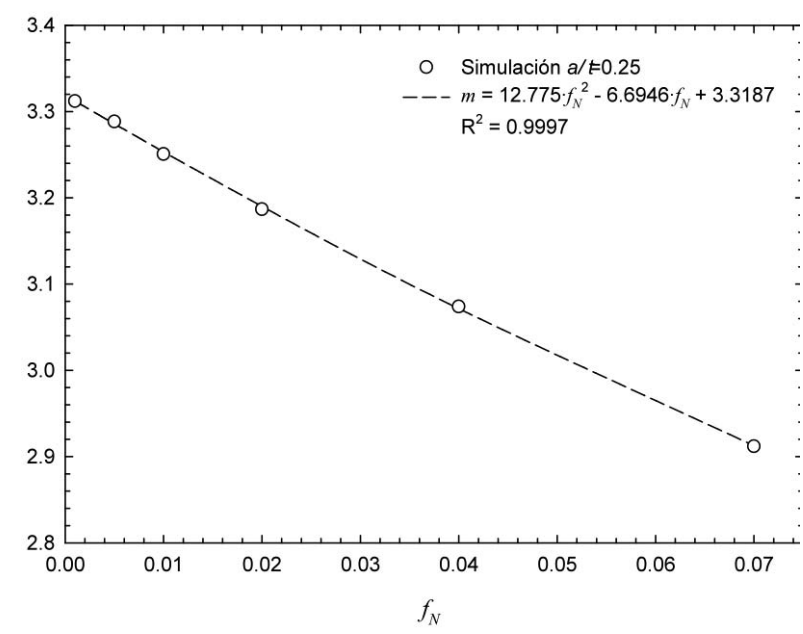

Figura 11. Variación de la pendiente $m$ en función del valor de $f_{N}$.

Figure 11. Slope variation versus $f_{N}$.

recogida la variación de la pendiente frente al valor de $f_{\mathrm{N}}$. Entrando en el gráfico con el valor de $m=3,27$ se obtiene el valor de $f_{N}=0,007$. Este valor de es el utilizado para proseguir con la metodología de identificación de parámetros.

\subsection{Determinación de $f_{F}$}

El inicio de propagación de fisura en la probeta, durante el ensayo, está ligado a la coalescencia de 
microhuecos a nivel macroscópico, es decir, que la fracción volumétrica de microhuecos ha alcanzado el valor de $f_{\mathrm{F}}$.

En el ensayo experimental es posible establecer el inicio de propagación, a partir de diferentes técnicas ${ }^{[30]}$. En el caso de las probetas ensayadas con relación $a / t=0,25$, se ha obtenido que el inicio de propagación se produce para un valor de desplazamiento del punzón entorno a 1,75 mm.

Por otro lado, se ha observado que el inicio de propagación de fisura se produce en la zona central de la probeta, en torno a la punta de fisura ${ }^{[30]}$. La figura 12 muestra la evolución de la fracción volumétrica de microhuecos en esta zona, donde comenzará el fallo de la probeta.

La identificación del valor de $f_{F}$ se puede realizar mediante la figura 12, en la que entrando con el dato del valor de desplazamiento del punzón en el inicio de propagación de fisura $(1,75 \mathrm{~mm})$ se obtiene el valor de la fracción volumétrica de microhuecos, en ese instante, para $f_{F}=0,007$, el cuál será el valor de $f_{\mathrm{F}}$, quedando $f_{\mathrm{F}}=0,065$.

\subsection{Determinación de $f_{\mathrm{c}}$}

Una vez determinado el valor de $f_{F}$ y alcanzado el punto de carga máxima, la forma de la curva, en la región IV, es gobernada por el valor de $f_{c}$; variando este valor, la velocidad de coalescencia de microhuecos se alterará. Se han realizado varias simulaciones modificando el parámetro para comprobar cuál se ajusta mejor a los datos experimentales. En la figura 13 se

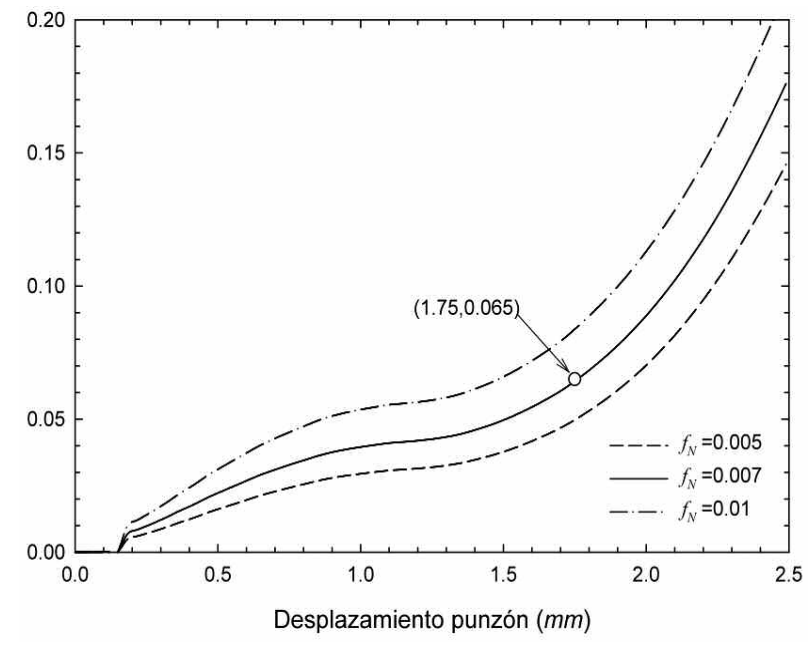

Figura 12. Porosidad acumulada en la zona central con diferentes valores de $f_{N}$.

Figure 12. Cumulative porosity in central zone with different $f_{N}$ values.

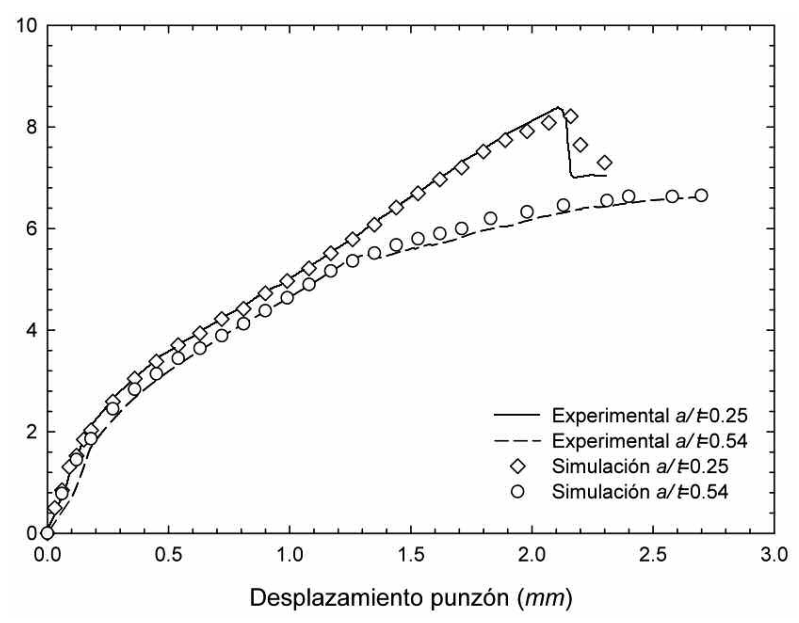

Figura 13. Comparación ensayo experimentalsimulación con $f_{N}, f_{c} \mathrm{y} f_{F}$.

Figure 13. Experimental test versus simulation with $f_{N}, f_{c}$ and $f_{F}$.

puede observar que, para $f_{c}=0,02$, se obtiene un buen ajuste de los mismos.

\section{CONCLUSIONES}

En la presente investigación se ha desarrollado una metodología para la identificación de los parámetros del modelo de daño de GTN, a partir de la curva carga-desplazamiento obtenida en el SPT sobre probeta prefisuradas con relación $a / t=0,25$.

La tabla III muestra los valores de dichos parámetros obtenidos para el acero 15,5 $\mathrm{PH}$, con los cuáles es posible simular correctamente otras geometrías de similar triaxialidad, como puede ser el caso de una probeta SPT prefisurada con una relación $a / t=0,54$ (Fig. 13). Se ha comprobado que los parámetros de material empiezan a tener influencia sobre la curva carga-desplazamiento, obtenida en el SPT, en las diferentes regiones en las que se puede dividir la curva permitiendo, así, su identificación a partir de la curva

Tabla III. Parámetros de daño del modelo de GTN para el acero $15,5 \mathrm{PH}$

Table III. Parameters of the GTN damage model for $15.5 \mathrm{PH}$ stainless steel

\begin{tabular}{cccccccc}
\hline$q_{1}$ & $q_{2}$ & $f_{0}$ & en & $s$ & $f_{N}$ & $f_{c}$ & $f_{F}$ \\
\hline 1,5 & 1 & 0 & 0,1 & 0,01 & 0,007 & 0,02 & 0,065
\end{tabular}


experimental. El procedimiento presentado puede hacerse extensible a otro tipo de materiales metálicos.

\section{Agradecimientos}

Los autores desean agradecer la financiación recibida del proyecto MCI Ref: MAT2008-06879-C0303/MAT.

\section{REFERENCIAS}

[1] J.M. Baik, J. Kameda y O. Back, Scr. Metall. Materialia 17 (1983) 1.443-1.447.

[2] X. Mao y H. Takahashi, J. Nucl. Mater. 150 (1987) 42-52.

[3] X. Mao, H. Takahashi y T. Kodaira, Mater. Sci. Eng. A I50 (1992) 231-236.

[4] T. Misawa, T. Adachi, M. Saito y Y. Hamaguchi, J. Nucl. Mater. 150 (1987) 194-202.

[5] T. Misawa, Y. Hamaguchi y M. Saito, J. Nucl. Mater. 155-157 (1988) 749-753.

[6] T. Misawa, S. Nagata, N. Aoki, J. Ishizaka y Y. Hamaguchi, J. Nucl. Mater. 169 (1989) 225 232.

[7] T. Misawa, K. Suzuki, M. Saito y Y. Hamaguchi, J. Nucl. Mater. 179-181 (1991) 421-424.

[8] T. Misawa, T. Ohtsuka, M. Seo y M. Saito, J. Nucl. Mater. 179-181 (1991) 611-614.

[9] S.H. Chi, J.H. Hong y I.S. Kim, Scr. Metall. Materialia 30 (1994) 1.521-1.525.

[10] M.L. Saucedo-Muñoz, S.C. Liu, T. Hashida, H. Takahashi y H. Nakajima, Cryogenics 41 (2001) 713-719.

[11] Y. Ruan, P. Spätig y M. Victoria, J. Nucl. Mater. 307-311 (2002) 236-239.

[12] E.N. Campitelli, P. Spätig, R. Bonadé, W. Hoffelner y M. Victoria, J. Nucl. Mater. 335 (2004) 366-378.

[13] M.A. Contreras, C. Rodríguez, F.J. Belzunce, y C. Betegón, Fatigue Fract. Eng. Mater. Struct. 31 (2008) 727-737.
[14] J. Ju, J. Jang y D. Kwon, Int. J. Press. Vessels Pip. 80 (2003) 221-228.

[15] I.I. Cuesta, J.M. Alegre y R. Lacalle, J. A. Álvarez y F.Gutiérrez-Solana, Anal. Mec. 25 (2008) 486-491.

[16] I.I. Cuesta, J.M. Alegre y P.M. Bravo, Anal.Mec. Fract. 26 (2009) 382-387.

[17] ASTME 8M, Annual Book or ASTM Standards, Vol. 3.01, American Society for Testing and Materials, (2003).

[18] W. Ramberg y W. R. Osgood, Technical Note 902, National Advisory Committee for Aeronautics, Washington DC, EE. UU., 1943, pp. 1-13.

[19] J. Autillo, M.A. Contreras, C. Betegón, C. Rodríguez y FJ. Belzunce, Anal. Mec. Fract. 23 (2006) 77-83.

[20] J. Besson, L. Devillers-Guerville y A. Pineau, Eng. Fract. Mech. 67 (2000) 169-190.

[21] A.L. Gurson, J. Eng. Mater. Tech. 99 (1977) 215.

[22] A.L. Gurson, Proc. Int Conf. Fracture (Ed) DMR Taplin, Oxford, Inglaterra, Pergamon2, 1977, pp. 357-364.

[23] V. Tvergaard, Adv. Appl. Mech. 27 (1990) 83151.

[24] V. Tvergaard y A. Needleman, Acta Metall. 32 (1985) 157-169.

[25] A. Needleman y V Tvergaard, Int. J. Fract. 49 (1991) 41-67.

[26] M. Abendroth y M. Kuna, Computational Mater. Sci. 28 (2003) 633-644.

[27] N. Benseddiq y A. Imad, Int. J. Press. Vessels Pip. 85 (2008) 219-227.

[28] A.G. Franklin, J. Iron Steel Inst. 207 (1969) 181-186.

[29] MSC.Marc, MSC Software Corporation, Volume A Theory and User Information 7 (2005) 152-154.

[30] I.I. Cuesta, Tesis Doctoral, Escuela Politécnica Superior, Universidad de Burgos, 2010.

[31] I.I. Cuesta, J.M. Alegre y R. Lacalle, Fat. Fract. Eng. Mater. Struct 33 (2010) 703-713. 